\title{
Factors Affecting Length of Stay in Pediatric Emergency Department in Zagazig University Hospital
}

Mohamed Mosaad Abohashem Ahmed Nassar, Beshir Adhallah Hassan, Hossam Fathi El-Saadany

Department of Pediatrics, Faculty of Medicine - Zagazig University

Corresponding author: Mohamed Mosaad Abohashem Ahmed Nassar, Mobile: (+20) 01000043784,

E-Mail: mmosaad521@gmail.com

\begin{abstract}
Background: Emergency medicine now is recognized as an essential part of public health service. As the services provided by emergency departments (EDs) increase and the management process becomes more complicated, patients stay in EDs for longer periods and EDs become more crowded. A number of studies have discussed the adverse impacts of ED crowding, which include prolonged waiting times, increased complications, and increased mortality.

Objective: The aim of this work was to analyze factors affecting length of stay (LOS) in the pediatric ED.

Patients and methods: This Cross-sectional study was conducted in the Pediatric Emergency Unit of Zagazig University Hospitals, from July 2019 until December 2019. Assuming that rate of admission of children in Emergency Department is 200 cases/month, so a comprehensive sample of 1200 children was included in the study.

Results: The age of the studied cases ranged from 1 month to 12 years with mean 3.62 years. Regarding sex, $53.4 \%$ were female. In our study, the $43.8 \%$ of patients were discharged from the ED in the current study. This study showed that $71.8 \%$ of the studied cases came to hospital from 9 am to $9 \mathrm{pm}$. There was no statistically significant difference between length of stay and sex. In the present study, mean value of length of stay (hours) was low among discharged patients $(0.20 \pm 0.59)$ than admitted patients $(0.51 \pm 0.58)$.

Conclusion: We concluded that several factors are associated with high average emergency department LOS. High LOS may lead to increases in expenditures and may have implications for patient safety, whereas certain organizational changes, communication improvement, and time management may have a positive effect on it. Keywords: Length of stay, Pediatric Emergency Department, Zagazig University Hospital.
\end{abstract}

\section{INTRODUCTION}

Emergency department (ED) staff face unique challenges such as treatment of patients arriving to the department with dynamic and unexpected states of illness, dealing with uncertainty regarding patient medical histories, and the need for time-dependent and triage-based decision-making. All of which are often accompanied by high financial costs to healthcare systems and a pressure to be economically efficient ${ }^{(\mathbf{1})}$.

According to the Institute of Medicine, one of the most common weak points of EDs is crowding, and it is important to understand the causes, effects, and prevention strategies for this. Overcrowding diminishes the capability of the EDs to manage and provide immediate access and stabilization for patients who have an emergency medical condition ${ }^{(2)}$. In a study analyzing crowding, researchers found three main factors contributing to it: input factors reflected sources and aspects of patient inflow, throughput factors reflected bottlenecks within the $\mathrm{ED}$, and output factors reflected bottlenecks in other parts of the healthcare system that can affect the $\mathrm{ED}^{(3)}$.

Excess inpatient LOS was defined as: exceeding the stated benchmark for the relevant diagnosis-related group. They found that compared with patients who stay in the ED for $4-8 \mathrm{~h}$; those who remain in the ED for $8-12 \mathrm{~h}$ are $20 \%$ more likely to stay in hospital longer than the national average for the relevant admission problem. Moreover, this number raised to $50 \%$ if emergency department LOS was greater than $12 \mathrm{~h}$. Another study examined one of the direct factors influencing emergency department LOS known as "access block," when hospital admission remains more than $8 \mathrm{~h}$ in the emergency department due to a lack of access to appropriate hospital inpatient beds ${ }^{(4)}$.

The study examined the relationship between access block in the ED, defined as the total time exceeding eight hours from a patient's initial arrival in the ED to transfer to another department and inpatient LOS. Results revealed that $7.7 \%$ of 11,906 patients experienced access block. In addition, the mean LOS was 4.9 days in those who experienced access block compared with 4.1 days in those who did not. Subgroup analysis showed that the effect of access block varied across severities of illness and diagnosis. For example, the mean LOS was 3.9 days in patients with cardiac diagnosis who experienced access block compared to 5.6 days in cardiac patients who did not experienced access block ${ }^{(4)}$.

The aim of this work was to analyze factors affecting the LOS in the pediatric ED. 


\section{PATIENTS AND METHODS}

This Cross-sectional study was conducted in the Pediatric Emergency Unit of Zagazig University Hospitals, from July 2019 until December 2019.

Sample size: Assuming that rate of admission of children in Emergency Department was 200 cases/month, so a comprehensive sample of 1200 children was included in the study.

Inclusion Criteria: Children in Pediatric Emergency Department in Zagazig University Hospitals, cardiac, renal, chest, neurological and GIT diseases.

Exclusion Criteria: Pediatric surgery and neonates.

All patients subjected to the following: History: Complete history taking (from the relatives). Examination: Complete clinical and physical examination. Investigation: Complete blood count (CBC), ALT-AST, S. urea, S. create, Random blood sugar (RBS), ABG, S. Electrolytes, Imaging (X-ray, ultrasonography and CT), Electrocardiograph (ECG), Echo, and Monitoring (HR, O2 saturation, RR and blood pressure).

\section{Data Collection:}

1- Time of registration.

2- Time of nursing.

3- Time of physician assessment.

4- Time of medical decision making (discharge vs. admittance).

5- Use of specialty consultation and ancillary services, as well as time of departure. Admitted patients were considered to have departed from the ED until they physically transported out of the ED to the hospital inpatient ward or another patient care facility (5).

Observers recorded in addition to time, any relevant aspect of the process of care during the patients stay in the ED that may have effect on ED LOS.

Ethical approval: The study protocol was approved by Ethics Committee, Faculty of Medicine, Zagazig University.

An informed consent from parents was provided after explanation of the nature and the purpose of the investigations to the parents prior to participation in the study.

\section{Statistical Analysis}

The data were coded, entered and processed on computer using Statistical package for social science (SPSS) version 24. The results were represented in tabular and diagrammatic forms then interpreted. Mean, standard deviation, range, frequency, and percentage were use as descriptive statistics. Chi-Square test $\mathrm{X}^{2}$ was used to test the association variables for categorical data. Student's t-test was used to assess the statistical significance of the difference between two population means in a study involving independent samples. ANOVA (F test) for normally quantitative variables, to compare between more than two groups, and Post Hoc test (LSD) for pairwise comparisons. $\mathrm{P}>0.05$ : Non-significant. $\mathrm{P} \leq 0.05$ : Significant.

\section{RESULTS}

Table (1): Demographic data, General examination and Length of stay and arrival time of the studied group

\begin{tabular}{||c|c|c||}
\hline \multicolumn{1}{|c|}{ Variable } & \multicolumn{2}{|c|}{$(\mathbf{n}=1200)$} \\
\hline \hline $\begin{array}{c}\text { Age: (year) } \\
\text { Mean } \pm \text { SD } \\
\text { Median }\end{array}$ & \multicolumn{2}{|c|}{$3.62 \pm 2.44$} \\
3
\end{tabular}

Sd: Standard deviation

This table showed that the age of the studied cases ranged from 6 months to 12 years with mean 3.62 years. Body weight ranged from 6 to $60 \mathrm{~K}$ with mean $20.82 \mathrm{Kg}$. Regarding sex, $53.4 \%$ were female. This table showed that most frequent findings among the studied group were fever and respiratory findings (66.3\% \& $50.7 \%$ respectively). Moreover, this table showed that $71.8 \%$ of the studied cases came to hospital from $12 \mathrm{pm}$ to $12 \mathrm{am}$. Also length 
of stay ranged from 0 to 2 hours with mean 0.48

hour.

Table (2): Relation between length of stay and sex

\begin{tabular}{|c|c|c|c|c|}
\hline \multicolumn{2}{|c|}{ Variable } & Length of stay & \multirow{2}{*}{ MW } & \multirow{2}{*}{ P } \\
\cline { 3 - 3 } & Mean \pm SD & \multirow{2}{*}{0.21} & $0.83 \mathrm{NS}$ \\
\hline \hline \multirow{2}{*}{ Sex } & Male & $\begin{array}{l}0.49 \pm 0.49 \\
0.48 \pm 0.64\end{array}$ & 0.21 & \\
\hline
\end{tabular}

Sd: Standard deviation MW: Mann Whitney test

NS: Non significant $(p>0.05)$

There was no statistically significant difference between male and female in length of stay.

Table (3): Relation between length of stay and cardiac finding

\begin{tabular}{|c|c|c|c|c|c||}
\hline \multicolumn{2}{|c|}{ Variable } & \multirow{2}{*}{ N } & Length of stay & \multirow{2}{*}{ KW } & \multirow{2}{*}{ P } \\
\cline { 4 - 4 } & & Mean \pm SD & & \\
\hline \hline \multirow{4}{*}{ Cardiac } & HF & 32 & $0.17 \pm 0.14$ & & \\
& Arrhythmia & 18 & $0.29 \pm 0.26$ & \multirow{3}{*}{9.92} & $<0.001 * *$ \\
& Other & 35 & $0.21 \pm 0.15$ & & \\
& No & 1115 & $0.57 \pm 0.37$ & & \\
\hline
\end{tabular}

Sd: Standard deviation KW: Kruskal Wallis test

$* *$ : Highly significant $(\mathrm{p}<0.01)$

There was statistically significant decrease in length of stay among patients with cardiac findings especially HF compared to cases without.

Table (4): Relation between length of stay and respiratory finding

\begin{tabular}{|c|c|c|c|c|c|}
\hline \multirow{2}{*}{\multicolumn{2}{|c|}{ Variable }} & \multirow{2}{*}{$\mathbf{N}$} & Length of stay & \multirow{2}{*}{ KW } & \multirow{2}{*}{$\mathbf{P}$} \\
\hline & & & Mean \pm SD & & \\
\hline Respiratory & $\begin{array}{c}\text { Bronchitis } \\
\text { Pneumonia } \\
\text { F B } \\
\text { Other } \\
\text { No }\end{array}$ & $\begin{array}{c}209 \\
165 \\
10 \\
242 \\
574\end{array}$ & $\begin{array}{l}0.34 \pm 0.24 \\
0.18 \pm 0.13 \\
0.16 \pm 0.12 \\
0.28 \pm 0.19 \\
0.55 \pm 0.48\end{array}$ & 12.34 & $0.009 * *$ \\
\hline
\end{tabular}

Sd: Standard deviation KW: Kruskal Wallis test

$* *$ : Highly significant $(\mathrm{p}<0.01)$

There were statistically significant decrease in length of stay among patients with respiratory findings especially F B and pneumonia compared to cases without.

Table (5): Relation between length of stay and Neurological finding

\begin{tabular}{|c|c|c|c|c|c|}
\hline \multirow{2}{*}{\multicolumn{2}{|c|}{ Variable }} & \multirow{2}{*}{$\mathbf{N}$} & Length of stay & \multirow{2}{*}{ KW } & \multirow{2}{*}{$\mathbf{P}$} \\
\hline & & & Mean \pm SD & & \\
\hline Neurological & $\begin{array}{c}\text { Febrile convulsion } \\
\text { G.B.S } \\
\text { C.S infection } \\
\text { Other } \\
\text { No }\end{array}$ & $\begin{array}{c}51 \\
10 \\
53 \\
51 \\
1035 \\
\end{array}$ & $\begin{array}{l}0.22 \pm 0.34 \\
0.27 \pm 0.24 \\
0.26 \pm 0.22 \\
0.31 \pm 0.19 \\
0.57 \pm 0.32\end{array}$ & 11.23 & $0.01 *$ \\
\hline
\end{tabular}

Sd: Standard deviation KW: Kruskal Wallis test $\quad$ *: Significant $(\mathrm{p}<0.01)$

There were statistically significant decrease in length of stay among patients with neurological findings especially febrile convulsion compared to cases without.

Table (6): Relation between length of stay and fate

\begin{tabular}{|c|c|c|c|c|c|}
\hline \multirow{2}{*}{\multicolumn{2}{|c|}{ Variable }} & \multirow{2}{*}{$\mathbf{N}$} & Length of stay & \multirow{2}{*}{ KW } & \multirow{2}{*}{$\mathbf{P}$} \\
\hline & & & Mean \pm SD & & \\
\hline Fate & $\begin{array}{c}\text { Discharged } \\
\text { Referred } \\
\text { Admitted } \\
\text { Died }\end{array}$ & $\begin{array}{c}576 \\
38 \\
574 \\
12\end{array}$ & $\begin{array}{c}0.75 \pm 0.59 \\
0.51 \pm 0.48 \\
0.20 \pm 0.28 \\
0.12 \pm 0.09\end{array}$ & 10.06 & $<0.001 * *$ \\
\hline
\end{tabular}

Sd: Standard deviation KW: Kruskal Wallis test

$* *$ : Highly significant $(\mathrm{p}<0.01)$ 
There were statistically significant decrease in length of stay among patients died and admitted compared to discharged or referred.

Table (7): Relation between length of stay and Arrival time

\begin{tabular}{|c|c|c|c|c|c|}
\hline \multirow{2}{*}{\multicolumn{2}{|c|}{ Variable }} & \multirow{2}{*}{$\mathbf{N}$} & Length of stay & \multirow{2}{*}{ MW } & \multirow{2}{*}{$\mathbf{P}$} \\
\hline & & & Mean \pm SD & & \\
\hline Arrival time & $\begin{array}{l}\text { Pm } \\
\text { Am }\end{array}$ & $\begin{array}{l}338 \\
862\end{array}$ & $\begin{array}{l}0.46 \pm 0.55 \\
0.61 \pm 0.63\end{array}$ & 2.18 & $0.04 *$ \\
\hline
\end{tabular}

Sd: Standard deviation MW: Mann Whitney test

*: Significant $(\mathrm{p}<0.05)$

There was statistically significant decrease in length of stay among cases arrived at pm than am.

Table (8): Correlation between length of stay and age, weight and vital signs among the studied group

\begin{tabular}{|c|c|c|}
\hline \multicolumn{2}{|c|}{ Variable } & \multirow{2}{*}{$\begin{array}{c}\begin{array}{c}\text { Length of stay } \\
(\mathrm{n}=1200)\end{array} \\
0.37\end{array}$} \\
\hline \multirow{2}{*}{ Age (years) } & $\mathbf{r}$ & \\
\hline & $\overline{\mathbf{P}}$ & $0.02 *$ \\
\hline \multirow{2}{*}{ Weight (kg) } & $\mathbf{r}$ & 0.012 \\
\hline & $\overline{\mathbf{P}}$ & 0.68 \\
\hline \multirow{2}{*}{ O2 sat. } & $\mathbf{r}$ & 0.50 \\
\hline & $\mathbf{P}$ & $<0.001 * *$ \\
\hline \multirow{2}{*}{ HR } & $\mathbf{r}$ & -0.47 \\
\hline & $\mathbf{P}$ & $0.008 * *$ \\
\hline \multirow{2}{*}{$\mathbf{R R}$} & $\mathbf{r}$ & 0.14 \\
\hline & $\mathbf{P}$ & $0.16 \mathrm{NS}$ \\
\hline \multirow{2}{*}{ SBP } & $\mathbf{r}$ & 0.31 \\
\hline & $\mathbf{P}$ & $0.04 *$ \\
\hline \multirow{2}{*}{ DBP } & $\mathbf{r}$ & 0.38 \\
\hline & $\mathbf{P}$ & $0.03 *$ \\
\hline \multirow{2}{*}{ GCS } & $\mathbf{r}$ & 0.48 \\
\hline & $\mathbf{P}$ & $0.003 * *$ \\
\hline
\end{tabular}

This table showed that there was statistical significance positive correlation between length of stay and age, $\mathrm{O}_{2}$ saturation, BP and GCS and there was statistically significant negative correlation between length of stay and heart rate.

\section{DISCUSSION}

This study was cross-sectional study, which was conducted in the Pediatric Emergency Unit of Zagazig University Hospitals on 1200 children.

This study showed that, the age of the studied cases ranged from 1 month to 12 years with a mean of 3.62 years. This is lower than Hofer and Saurenmann (6) who aimed to identify and quantify variables, which affect ED-LOS. Patients admitted to the pediatric ED of a large regional Swiss hospital during a 1-year period were analyzed for LOS (in minutes). Predictor variables included patient-associated parameters (time of admission and discharge, ED occupancy, triage score, diagnosis, and demographic data) and external factors (weekday, time, and season). A total of 4885 visits were included. They found that, among the selected sample, females accounted for 2142 of the visits (43.9\%). This study showed that most frequent findings among the studied group were fever and respiratory findings $(66.3 \% \& 50.7 \%$ respectively). This agrees with Niska et al. ${ }^{(7)}$ who made a study in the USA and found that, the leading reasons for the pediatric ED among children (aged under 15 years) were fever and cough. In Li et al. ${ }^{(8)}$ study, fever accounted for 17,741 (61.1\%) of the pediatric ED patient visits. In the present study, length of stay ranged from 0 to 6 hours with mean 0.48 minute. Harris and Hostetler ${ }^{(9)}$ revealed the overall mean LOS of 2.6 hours. In our study, $43.8 \%$ of patients were discharged from the ED in the current study. Compared with previous studies, the ED discharge rate was $71 \%{ }^{(10)}$.

This study showed that, $71.8 \%$ of the studied cases came to hospital from 9 am to $9 \mathrm{pm}$. This agrees with Bashkin et al. ${ }^{(5)}$ who aimed to examine LOS in 
the emergency department and explored the main factors that influence LOS and cause delay in patient care. Observations of 105 patients were performed over a 3-month period at the emergency room of a community urban hospital. They found that $52 \%$ were registered during the morning shift and $48 \%$ during the evening shift. Another predictive factor for prolonged ED-LOS was admission in the morning and around noon. While, fewer patients consulted the ED in the morning compared to evening hours and ED occupancy increased over the day with a peak period between 4 and 8 p.m., the number of patients subject to prolonged LOS was highest before noon. Various authors assume that variations in staffing and organizational processes may contribute to reduced throughput in the morning hours ${ }^{(\mathbf{1 1})}$.

This study showed that, there was no statistically significant difference between length of stay and sex. This agrees with Dada and Sule ${ }^{(\mathbf{1 2})}$ who found no statistically significant difference between length of stay and sex. This study showed that, there was statistical significance positive correlation between length of stay and age. Age is another factor that was found to be related to increase ED LOS across different nations ${ }^{(\mathbf{1 3})}$.

This study showed that, there were statistically significant increase in length of stay among arrival time at am than pm.

Another study by Hofer and Saurenmann (6) found another predictive factor for prolonged ED-LOS was admission in the morning and around noon. While fewer patients consulted the ED in the morning compared to evening hours and ED occupancy increased over the day with a peak period between 4 and 8 p.m., the number of patients subject to prolonged LOS was highest before noon.

This study showed that, there were statistically significant increase in length of stay among patients with respiratory findings than without. This agrees with Dada and Sule ${ }^{(12)}$ who aimed to determine whether the addition of a case manager and a physician advisor to the observation unit would decrease the LOS of patients' observation. Their retrospective, observational study for patients' observation was conducted in 2017. At a tertiary-care medium-sized urban community hospital, the LOS for all patients' observation. They found that, patients with respiratory findings had significantly longer LOS.

This study showed that there was statistically significant increase in length of stay among patients with cardiac findings than without. This agrees with Leykum et al. ${ }^{(14)}$ who found in their retrospective analysis that LOS for cardiac patients increased than without ( 2.4 days to 2.2 days, $p=0.05$ )

In the present study, mean value of length of stay (hours) was low among discharged patients $(0.20$ $\pm 0.59)$ than admitted patients $(0.51 \pm 0.58)$. This is in accordance with the study done by Chaou $\boldsymbol{e t}$ al. ${ }^{(15)}$ who found that for the admitted patients, the median LOS was much longer than for the discharged patients. This might be explained by the fact that the priority for admission within a specific medical specialty is ranked according to both the severity of the disease and the arrival time and that ICU waiting times are generally shorter than ward waiting times. It is clear from the previous literature that the LOS of the admitted patients is determined not only by ED or patient factors but also by hospital-level determinants such as hospital occupancy, admission-discharge ratio and the daily hospital and ICU census ${ }^{(16)}$.

Thus, some of the strategies developed to improve ED LOS for admission patients, such as creating specialized acute medical admission wards, increasing ICU capacity, arranging admission immediately after evaluation, or developing diseasespecific protocols, may be very different from those for discharged patients ${ }^{(\mathbf{1 7 )}}$.

In the present study, mean value of length of stay (hours) was high among referred patients $(0.75 \pm$ 0.076) than other patients. Bashkin et al. ${ }^{(5)}$ in their study revealed that problems in continuum-of-care were found to affect ED LOS mainly among the admitted patients group. The process-from deciding to admit a patient until the patient was actually transferred from the ED to an inpatient hospital ward took $43 \%$ of the total ED LOS of admitted patients. Analysis of the admitting process using Ishikawa diagram revealed several possible explanations to the prolonged waiting times, one of them being deficient communication.

Physician referral was a strong predictor of prolonged ED-LOS in our study. The reason was most likely the higher complexity and severity of these cases, which were referred to the ED after a visit with a community-based pediatrician or family doctor. This association is reflected by lower levels of triage in referred children. Also, admission diagnoses made by primary care physicians and specialists were subject to certain inaccuracies, which may lead to increased EDLOS, a fact reported by Bernhard et al. ${ }^{(18)}$ in a German adult ED of a tertiary care center. Furthermore, Gaucher et al. ${ }^{(19)}$ noted that patients who were referred by a physician are less likely to leave without being seen, which may prolong ED visit time.

The literature suggests that lack of continuumof-care in the process of patient admissions is derived from deficient communication among medical staff. Kripalani et al. ${ }^{(20)}$ and our study found that this was associated with, and might led to, prolonged ED LOS. Continuity of patient care is based on the effective transfer of information between medical staff members. A study that examined the handoff process between ED medical staff and Intensive Care unit (ICU) medical staff revealed that there was no structured and consistent approach to how handovers actually occurred. Moreover, nurses from both ED and 
ICU lacked clarity as to when the actual handover process began. Nurses from both settings recognized the importance of the information given and received during handover and deemed it to have an important role in influencing the quality and continuity of care (21).

In a study examining the perceptions of ED physicians and hospitalizing physicians regarding handoff communication of patients transferred from ED to inpatient settings, researchers found that physicians perceived handoff communication as characterized by ambiguity about patients' conditions and treatment. They found that poor communication practices and conflicting communication expectations presented barriers that exacerbated physicians' information ambiguity. They noted that ED physicians and receiving inpatient physicians had different expectations about handoffs and those expectations influenced their interactions in ways that could result in communication breakdowns. Hospitalizing physicians expect ED physicians to produce definitive diagnoses, and admissions are delayed until confirmatory test results are provided, whereas emergency physicians might believe that their professional opinions are being questioned ${ }^{(22)}$.

\section{CONCLUSION}

We concluded that several factors are associated with high average emergency department LOS. High LOS may lead to increases in expenditures and may have implications for patient safety, whereas certain organizational changes, communication improvement, and time management may have a positive effect on it.

\section{REFERENCES}

1. McFetridge B, Gillespie M, Goode D et al. (2007): An exploration of the handover process of critically ill patients between nursing staff from the emergency department and the intensive care unit. Nurs Crit Care, 12: 261-9.

2. Handel D, Ginde A, Raja A et al. (2010): Implementation of crowding solutions from the American College of Emergency Physicians Task Force Report on Boarding. Int J Emerg Med., 3 (4): 279-86.

3. Hoot N, Aronsky D (2008): Review of emergency department crowding: Causes, effects, and solutions. Ann Emerg Med., 52: 127-36.

4. James A, Nathan J, Jacqui C et al. (2019), The relationship between time to analgesic administration and emergency department length of stay:A retrospective review. Journal of Advanced Nursing, 76: 1190-93.

5. Bashkin O, Caspi S, Haligoa R et al. (2015): Organizational factors affecting length of stay in the emergency department: initial observational study. Israel Journal of Health Policy Research, 4: 38-40.

6. Hofer K, Saurenmann R (2017): Parameters affecting length of stay in a pediatric emergency department: a retrospective observational study. Eur J Pediatr., 176: 591-598.

7. Niska R, Bhuiya F, Xu J (2010): National Hospital Ambulatory Medical Care Survey: 2007 emergency department summary. Natl Health Stat Rep., 26: 1-31.

8. Li S, Chiu N, Kung W et al. (2013): Factors Affecting Length of Stay in the Pediatric Emergency Department. Pediatrics \& Neonatology, 54 (3): 179-187.

9. Harris N, Hostetler M (2002): Parental expectations of care and charges in a tertiary care pediatric ED. Am J Emerg Med., 20: 601-3.

10. Vermeulen M, Ray J, Bell C et al. (2009): Disequilibrium between admitted and discharged hospitalized patients affects emergency department length of stay. Ann Emerg Med., 54: 794-804.

11.Karaca Z, Wong H, Mutter R (2012): Duration of patients' visits to the hospital emergency department. BMC Emerg Med., 12: 15-19.

12.Dada R, Sule A (2019): Factors Affecting Length of Stay for Observation Patients. Cureus., 11 (4): 4547-4552.

13. Capuano F, Lot A, Sagnes-Raffy C (2015): Factors associated with the length of stay of patients discharged from emergency department in France. Eur J Emerg Med., 22: 92-98.

14.Leykum L, Huerta V, Mortensen E (2010): Implementation of a hospitalist-run observation unit and impact on length of stay (LOS): a brief report. J Hosp Med., 5: 2-5.

15. Chaou C, Chiu T, Yen A et al. (2016): Analyzing Factors Affecting Emergency Department Length of Stay Using a Competing Risk-accelerated Failure Time Model. Medicine, 95 (14): 3263-68.

16.Venkatesh A, Curley D, Chang Y (2015): Communication of vital signs at emergency department handoff: opportunities for improvement. Ann Emerg Med., 66: 125-130.

17.Lo S, Choi K, Wong E et al. (2014): Effectiveness of Emergency Medicine Wards in reducing length of stay and overcrowding in emergency departments. Int Emerg Nurs., 22: 116-120.

18. Bernhard M, Raatz C, Zahn P (2013): Validity of admission diagnoses as process-driving criteria: influence on length of stay and consultation rate in emergency departments. Anaesthesist, 62: 617-623.

19. Gaucher N, Bailey B, Gravel J (2011): Who are the children leaving the emergency department without being seen by a physician? Acad Emerg Med., 18: 152-157.

20. Kripalani S, LeFevre F, Phillips C et al. (2007): Deficits in Communication and information transfer between hospital-based and primary care physicians: implications for patient safety and continuity of care. JAMA., 297 (8): 831-41.

21.McGarry J, Krall S, McLaughlin T (2010): Impact of resident physicians on emergency department throughput. West J Emerg Med., 11: 333-335.

22. Apker J, Mallak L, Gibson S (2007): Communication in the "gray zone": perceptions about emergency physician hospitalist handoffs and patient safety. Acad Emerg Med., 14: 884-94. 